Eff i ci ent Shape and Topol ogy Opt i mi zat i on Based on Sensi ti vi ty Anal ysi s for Opt i cal Wavegui de Devi ces Uti I i zi ng Ful I-Vect or i al BPM

\begin{tabular}{|l|l|}
\hline 著者 & $\begin{array}{l}\text { I GUCH Aki to, TSUJ I Yasuhi de, YASUl Takashi , } \\
\text { H RAYAMA Koi chi }\end{array}$ \\
\hline $\begin{array}{l}\text { j our nal or } \\
\text { publ i cat i on t i t l e }\end{array}$ & JOURNAL OF LI GHTMAVE TECHNOLOGY \\
\hline vol une & 38 \\
\hline number & 8 \\
\hline page r ange & 2328 2335 \\
\hline year & 2020 \\
\hline URL & ht t p: //hdl . handl e. net /10258/00010267 \\
\hline
\end{tabular}




\title{
Efficient Shape and Topology Optimization Based on Sensitivity Analysis for Optical Waveguide Devices Utilizing Full-Vectorial BPM
}

\author{
Akito Iguchi, Member, IEEE, Yasuhide Tsuji, Senior Member, IEEE, Member, OSA, \\ Takashi Yasui, Member, IEEE, Member, OSA, and Koichi Hirayama, Senior Member, IEEE
}

\begin{abstract}
We present a new design approach of shape and topology optimization utilizing the full-vectorial beam propagation method (FV-BPM) so as to achieve efficient design for highrefractive-index contrast optical waveguides and devices based on gradual mode-evolution. In our design approach, material distribution is represented via normalized density parameters, and these design parameters are updated with sensitivity computed based on an adjoint variable method (AVM). The sensitivity analysis method and the design procedure are offered in the specific case that the density method, the AVM, and the FVBPM based on an alternative direction implicit method (AIDM) are employed. The applicability of our design approach is numerically studied by designing high-refractive-index contrast photonic components which induce polarization rotation: a TMOto-TE1 mode order converter and a polarization rotator. The results of the design examples indicate that our approach has potential to be suitable for efficient initial design of optical devices based on gradual mode-evolution.
\end{abstract}

Index Terms-Topology optimization, Adjoint variable method, Alternating direction implicit method, Beam propagation method, Optical waveguide device.

\section{INTRODUCTION}

$\mathbf{S}$ TRUCTURAL optimization using numerical simulation is widely employed techniques for design of optical circuit components used in optical communication networks. In particular, shape and topology optimization have attracted attention due to its potential to enhance device performance more highly than ever. In topology optimization, the aim is to find material distribution itself which fulfills the performance requirements. There are so many design variables in shape and topology optimization that the results of these optimization can offer novel concept of photonic devices with smaller footprint and higher performance. So far, it has been demonstrated that compact photonic components in planner lightwave circuit (PLC) or silicon on insulator (SOI) platform can be designed by shape or topology optimization [1]-[10].

In the design problems calling for 3D full-vectorial (FV) analysis, a great deal of computational cost is required because numerical analysis is iterated many times in the optimization

This work is supported by JSPS KAKENHI Grant Number JP 19J14709.

A. Iguchi and Y. Tsuji are with the Division of Information and Electronic Engineering, Muroran Institute of Technology, Muroran, 050-8585 Japan (email: iguchia@mmm.muroran-it.ac.jp; y-tsuji@mmm.muroran-it.ac.jp).

T. Yasui and K. Hirayama are with the Division of Electrical and Electronic Engineering, Kitami Institute of Technology, Kitami 090-8507, Japan (e-mail: yasui@mail.kitami-it.ac.jp; hirakc@mail.kitami-it.ac.jp).

Manuscript received Month XX, 2019; revised Month XX, 2019. process. As a numerical analysis method, the finite element method (FEM) [1]-[7], the finite difference time domain (FDTD) method [8], [9], or its frequency domain (FDFD) method [10] is widely applied to topology optimization, however, they require large computational effort in particular 3DFV problems. The beam propagation method (BPM) [11]-[15] is an efficient, useful technique for longitudinally varying optical waveguides. The BPM can greatly reduce computational cost because of a slowly varying envelope approximation (SVEA) which allows large mesh size in the longitudinal direction. Although the BPM does not offer exact solution due to the SVEA, this method can estimate wave propagation to some extent even in high-index-contrast waveguides as long as the variation of refractive index distribution is adiabatic enough in the propagation direction. The shape and topology optimization with the BPM has potential to be suitable for the initial design, where the accuracy does not count primarily, of high-index-contrast photonic components based on gradual mode evolution. In the authors' previous works [16], [17], we have already presented the topology optimal design approach with the BPM for 2D and 3D semi-vectorial design problems, and we have demonstrated its applicability for lowindex-contrast waveguides. The 3D-FV analysis is necessary for relatively high-index-contrast (high- $\Delta$ ) waveguide devices. Above all, it is a must to design devices which induce polarization rotation, such as polarization rotator (PR) [18][21], and polarization beam splitter [22]-[28].

In this paper, we adapt the topology optimal design approach reported in [17] so as to deal with the 3D-FV problems, and present shape and topology optimal design with FV-BPM. We verify the applicability of our design approach applying it to the design problems of a $\mathrm{TM}_{0}$-to- $\mathrm{TE}_{1}$ mode converter and a PR in the silicon nitride platform. We consider in this study the case that a finite difference (FD) scheme is employed to descritize the transverse direction, and an alternative direction implicit method (ADIM) is utilized for efficient FVFD-BPM analysis. As a way to express material distribution, we employ a density method. In this method, normalized density parameters are mapped in design region and refractive index distribution is expressed via density distribution. Since there are a large number of design parameters in topology optimization, these parameters are usually updated by a sequential (non)linear programming, or gradient methods with sensitivity of device performance. Sizing optimization based on sensitivity analysis with FV-BPM has already reported in [29] and a 
general way of sensitivity analysis is formulated, however, this report is not aimed at shape or topology optimization. We will describe the way of sensitivity analysis in the specific case of using the FV-BPM and the density method.

In section II, we review the FVFD-BPM based on ADIM, and describe present shape and topology optimal design approach, and the way of sensitivity analysis based on adjoint variable method (AVM) in the case of using the FVFDBPM and the density method. In section III, the application examples of a $\mathrm{TM}_{0}$-to- $\mathrm{TE}_{1}$ mode converter and a $\mathrm{PR}$ are shown to verify applicability of the present design method for high-index-contrast waveguide devices. In addition, we give some discussion on application range of our approach. Section IV is the conclusion.

\section{Shape And TOPOlOgy Optimization With ThE FV-BPM}

\section{A. The FVFD-BPM based on the ADIM}

First, we will review the formulation of FVFD-BPM based on ADIM. We consider a 3D optical waveguide which is composed of linear, isotropic materials. The 3D-FV wave equation is [13]

$$
\frac{\partial^{2}\left\{\Phi_{t}\right\}}{\partial z^{2}}+\left([P]+k_{0}^{2} n^{2}[I]\right)\left\{\Phi_{t}\right\}=0
$$

with

$$
\begin{aligned}
\left\{\Phi_{t}\right\} & =\left\{\begin{array}{l}
\Phi_{x} \\
\Phi_{y}
\end{array}\right\} \\
{[P] } & =\left[\begin{array}{cc}
A_{x x}+A_{y y} & C_{x y} \\
C_{y x} & B_{x x}+B_{y y}
\end{array}\right]
\end{aligned}
$$

where $\Phi_{\alpha}(\alpha \in\{x, y\}, \Phi \in\{E, H\})$ is a transverse component of electromagnetic field, $k_{0}$ is a wavenumber in vacuum, $n$ is a refractive index, and $[I]$ is an identity matrix. $A_{\alpha \alpha}, B_{\alpha \alpha}$, and $C_{\alpha \beta}[(\alpha, \beta) \in\{(x, y),(y, x)\}]$ are differential operators defined by

$$
\begin{aligned}
A_{x x} \Phi_{x} & =\frac{\partial}{\partial x}\left\{p \frac{\partial\left(p^{-1} \Phi_{x}\right)}{\partial x}\right\} \\
A_{y y} \Phi_{x} & =q \frac{\partial}{\partial y}\left\{q^{-1} \frac{\partial \Phi_{x}}{\partial y}\right\} \\
B_{x x} \Phi_{y} & =q \frac{\partial}{\partial x}\left\{q^{-1} \frac{\partial \Phi_{y}}{\partial x}\right\} \\
B_{y y} \Phi_{y} & =\frac{\partial}{\partial y}\left\{p \frac{\partial\left(p^{-1} \Phi_{y}\right)}{\partial y}\right\} \\
C_{\alpha \beta} E_{\beta} & =\frac{\partial}{\partial \alpha}\left\{\frac{1}{n^{2}} \frac{\partial\left(n^{2} E_{\beta}\right)}{\partial \beta}-\frac{\partial E_{\beta}}{\partial \beta}\right\} \\
C_{\alpha \beta} H_{\beta} & =\frac{\partial H}{\partial \beta \partial \alpha}-n^{2} \frac{\partial}{\partial \beta}\left\{\frac{1}{n^{2}} \frac{\partial H_{\beta}}{\partial \alpha}\right\}
\end{aligned}
$$

where $p=1 / n^{2}, q=1$ for $\Phi=E$, or $p=1, q=n^{2}$ for $\Phi=H$. In this study, the transversal differential operator, $A_{\alpha \alpha}, B_{\alpha \alpha}$, and the cross coupling term, $C_{\alpha \beta}$, are approximated by Stern's method [30], [31], and six-point FD formula described in [32], [33], respectively. The computational window is terminated by a perfectly matched layer (PML) based on complex coordinate stretching [34]:

$$
\frac{\partial}{\partial \alpha} \rightarrow \frac{1}{s_{\alpha}} \frac{\partial}{\partial \alpha}
$$

where

$$
s_{\alpha}=1-j\left(\frac{d}{T_{\mathrm{PML}, \alpha}}\right)^{2} \tan \delta .
$$

$T_{\mathrm{PML}, \alpha}$ is thickness of the PML, $d$ is distance from an interface between computational window and the PML, and $\tan \delta$ is loss tangent. When the phase constant of traveling light-wave in $+z$-direction is in the vicinity of $k_{0} n_{0},\left\{\Phi_{t}\right\}$ can be described by

$$
\left\{\Phi_{t}\right\}=\left\{\phi_{t}\right\} \exp \left(-j k_{0} n_{0} z\right)
$$

with

$$
\left\{\phi_{t}\right\}=\left\{\begin{array}{c}
\phi_{x} \\
\phi_{y}
\end{array}\right\} \text {. }
$$

$\phi \in\{e, h\}$ is the slowly varying envelope amplitude with respect to $z$-direction. In the FV-BPM based on ADIM and an implicit Crank-Nicolson scheme [15], the output field from an optical component, $\left\{\phi_{t}\right\}_{N_{z}}$, can be computed by

$$
\left\{\phi_{t}\right\}_{N_{z}}=\prod_{l=0}^{N_{z}-1}\left(\left[\Gamma_{4}\right]^{-1}\left[\Gamma_{3}\right]\left[\Gamma_{2}\right]^{-1}\left[\Gamma_{1}\right]\right)_{l}\left\{\phi_{t}\right\}_{0}
$$

where $\left\{\phi_{t}\right\}_{k}=\left\{\phi_{t}(x, y, k \Delta z)\right\},\left[\Gamma_{a}\right]_{k}(a=1, \ldots, 4)$ are propagation operators at $k$-th cross section, and $\Delta z$ is step size in $z$-direction. Note that in (10), $\Pi$ is a reverse-order product:

$$
\prod_{l=0}^{N} U_{l} \equiv U_{N} U_{N-1} \cdots U_{1} U_{0}
$$

(10) can be computed very efficiently using Gaussian elimination in the tridiagonal matrix, known as the Thomas algorithm.

\section{B. Shape and topology optimization with FV-BPM}

In the density-based topology optimization, the material distribution is represented via the normalized density parameter [1]. The relative permittivity distribution in a certain design region, $n_{\Omega}^{2}\left(=\varepsilon_{r, \Omega}\right)$, can be expressed via normalized density distribution as follows:

$$
n_{\Omega}^{2}(x, z)=n_{\mathrm{cl}}^{2}+\left(n_{\mathrm{co}}^{2}-n_{\mathrm{cl}}^{2}\right) H\left(\rho_{\Omega}(x, z), m\right) .
$$

$n_{\mathrm{cl}}$ and $n_{\mathrm{co}}$ are the refractive indices of a cladding and a core material, respectively. We assume that in (11) core height is uniform in the design region as shown in Fig. 1. $H(\rho, m)$ $\in[0,1]$ is a Heaviside function with a transition region. The definition is described in [16]. $m$ is a penalty parameter which controls intermediate materials (gray materials) between the cladding (white) and the core (black) materials. $H(\rho,+\infty)$ means a complete Heaviside step function. The gray region is completely removed when $m \rightarrow+\infty$. Since the gray materials are not real, they should be removed in the final phase of optimization. However, device performance is not 


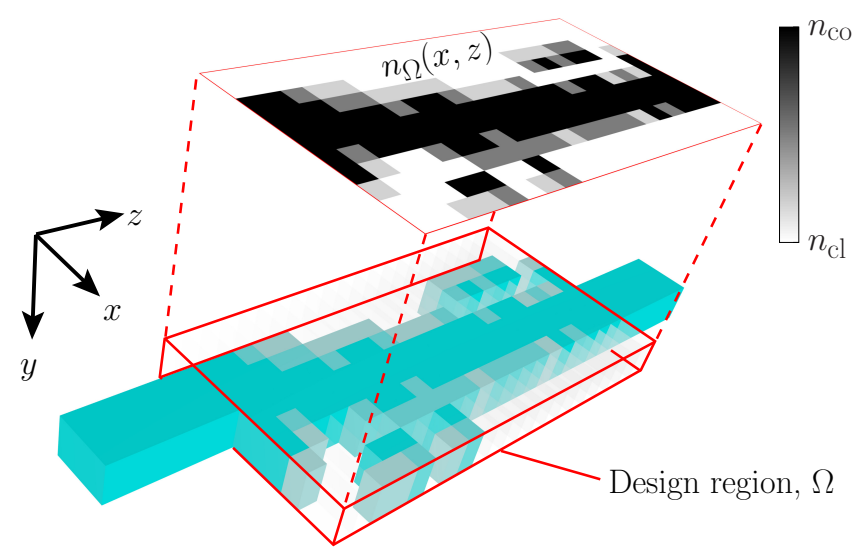

Fig. 1. 3D schematic of reflactive index distribution in (11). $z$ is longitudinal direction of an optical component. The layer width and depth are $x$ - and $y$ direction, respectively.

improved if $m$ is excessively large because the value of the sensitivity is zero in the non-gray region. In this study, $m$ is moderate small value in the initial phase of optimization, and we increase gradually the value toward the final phase. The procedure is described in detail later in this section. When the computational region is discretized by FD cells and the density parameters are allocated at each cell, (11) is rewritten by

$$
n_{i, k}^{2}=n_{\mathrm{cl}}^{2}+\left(n_{\mathrm{co}}^{2}-n_{\mathrm{cl}}^{2}\right) H\left(\rho_{i, k}, m\right)
$$

where $n_{i, k}^{2}=n_{\Omega}^{2}(i \Delta x, k \Delta z)$ and $\rho_{i, k}=\rho_{\Omega}(i \Delta x, k \Delta z)$ $[(i \Delta x, k \Delta z) \in \Omega]$. Shape and topology optimization can be carried out by solving an optimization problem of the density parameters.

Performance of optical waveguide components is usually represented by normalized power which is computed using an S-parameter, or normalized power $\left|S_{n 1, r}\right|^{2}$ where $S_{n 1, r}$ denotes an S-parameter related to an input modal field in port 1 (input port) and an $r$-th eigenmode field in port- $n$ (output port). In the case of our study, the sensitivities with respect to the density parameters $\left(\partial S_{n 1, r} / \partial \rho_{i, k}\right)$ have to be computed to update the density distribution so that the performance is improved. We assume an eigenmode field in port- $n$ is normalized by

$$
\iint_{D_{N_{z}}}\left(\psi_{y, n, r}^{*} \phi_{x, n, r}-\psi_{x, n, r}^{*} \phi_{y, n, r}\right) d x d y=1
$$

An S-parameter can be estimated by the following overlap integral:

$$
s_{n 1, r}=\iint_{D_{N_{z}}}\left(\psi_{y, n, r}^{*} \phi_{x, N_{z}}-\psi_{x, n, r}^{*} \phi_{y, N_{z}}\right) d x d y
$$

where $\left(\phi, \psi, s_{n 1, r}\right) \in\left\{\left(e, h, S_{n 1, r}\right),\left(h, e, S_{n 1, r}^{*}\right)\right\}, \phi_{\alpha, n, r}$ and $\psi_{\alpha, n, r}$ are $r$-th eigenmode fields in port- $n, D_{k}$ is a transverse computational window at $z=k \Delta z, \phi_{\alpha, N_{z}}$ is an output field at $z=N_{z} \Delta z$, and ${ }^{*}$ denotes complex conjugate.

Discretized form of (13) can be written by

$$
s_{n 1, r}=\left\{g_{n, r}\right\}^{\dagger}\left\{\phi_{t}\right\}_{N_{z}}
$$

with

$$
\begin{aligned}
\left\{g_{n, r}\right\} & =\Delta x \Delta y\left[\begin{array}{c}
\left\{\psi_{y, n, r}\right\} \\
-\left\{\psi_{x, n, r}\right\}
\end{array}\right] \\
\left\{\phi_{t}\right\}_{N_{z}} & =\left[\begin{array}{l}
\left\{\phi_{x}\right\}_{N_{z}} \\
\left\{\phi_{y}\right\}_{N_{z}}
\end{array}\right]
\end{aligned}
$$

where ${ }^{\dagger}$ denotes Hermitian transpose. Differentiating both side of (14) to $\rho_{i, k}$, we have

$$
\frac{\partial s_{n 1, r}}{\partial \rho_{i, k}}=\left\{g_{n, r}\right\}^{\dagger} \frac{\partial\left\{\phi_{t}\right\}_{N_{z}}}{\partial \rho_{i, k}} .
$$

In (15), we assume that input port is not in the design region. Substituting (10) into (15), we get

$$
\begin{aligned}
\frac{\partial s_{n 1, r}}{\partial \rho_{i, k}}= & -\left\{\lambda_{n, r, k+1 / 2}\right\}^{T} \frac{\partial\left[\Gamma_{4}\right]_{k}}{\partial \rho_{i, k}}\left\{\phi_{t}\right\}_{k+1} \\
& +\left\{\lambda_{n, r, k+1 / 2}\right\}^{T} \frac{\partial\left[\Gamma_{3}\right]_{k}}{\partial \rho_{i, k}}\left\{\phi_{t}\right\}_{k+\frac{1}{2}} \\
& -\left\{\lambda_{n, r, k}\right\}^{T} \frac{\partial\left[\Gamma_{2}\right]_{k}}{\partial \rho_{i, k}}\left\{\phi_{t}\right\}_{k+\frac{1}{2}} \\
& +\left\{\lambda_{n, r, k}\right\}^{T} \frac{\partial\left[\Gamma_{1}\right]_{k}}{\partial \rho_{i, k}}\left\{\phi_{t}\right\}_{k}
\end{aligned}
$$

where ${ }^{T}$ denotes transpose. $\left\{\lambda_{n, r, k+1 / 2}\right\}$ and $\left\{\lambda_{n, r, k}\right\}$ are adjoint vectors obtained by solving the following adjoint equations:

$$
\begin{gathered}
{\left[\Gamma_{4}\right]_{k}^{T}\left\{\lambda_{n, r, k+1 / 2}\right\}=} \\
\prod_{l=k+1}^{N_{z}-1}\left(\left[\Gamma_{1}\right]^{T}\left[\Gamma_{2}\right]^{-1^{T}}\left[\Gamma_{3}\right]^{T}\left[\Gamma_{4}\right]^{-1^{T}}\right)_{l}\left\{g_{n, r}\right\}^{*} \\
{\left[\Gamma_{2}\right]_{k}^{T}\left\{\lambda_{n, r, k}\right\}=\left[\Gamma_{3}\right]_{k}^{T}\left\{\lambda_{n, r, k+1 / 2}\right\} .}
\end{gathered}
$$

Since the refractive index at an FD cell, $n_{i, k}$, is differentiable, the derivative of propagation matrix in the ADIM, $\partial\left[\Gamma_{a}\right]_{k} / \partial \rho_{i, j}$, can be computed without using a central finite difference approximation.

In the sensitivity analysis in this study, we let

$$
\left\{g_{n, r}\right\} \rightarrow \Delta x \Delta y\left[\begin{array}{l}
\left\{\phi_{x, n, r}\right\} \\
\left\{\phi_{y, n, r}\right\}
\end{array}\right]
$$

and in (16), $\left\{\lambda_{n, r, k}\right\}$ and $\left\{\lambda_{n, r, k+1 / 2}\right\}$ are let to be

$$
\begin{aligned}
\left\{\lambda_{n, r, k+1 / 2}\right\} & \rightarrow\left[p_{k}\right]\left\{\lambda_{n, r, k+1 / 2}\right\} \\
\left\{\lambda_{n, r, k}\right\} & \rightarrow\left[p_{k}\right]\left\{\lambda_{n, r, k}\right\} \\
{\left[p_{k}\right] } & =\left\{\begin{array}{cc}
{[I]} & \text { for } \phi=e \\
{\left[1 / n_{k}^{2}\right]} & \text { for } \phi=h
\end{array}\right.
\end{aligned}
$$

for simple implementation, where $\left[1 / n_{k}^{2}\right]$ is a diagonal matrix composed of cross sectional relative permittivity distribution, $\left\{1 / n_{k}^{2}\right\}$. In addition, we employ the following $3 \times 3$ simple moving average filter in order to avoid complex profile, such as a checkerboard pattern.

$$
\left(\frac{\partial f}{\partial \rho_{i, k}}\right)_{\text {filtered }} \leftarrow \sum_{\xi=-1}^{1} \sum_{\eta=-1}^{1} \frac{1}{9} \frac{\partial f}{\partial \rho_{i+\xi, k+\eta}}
$$




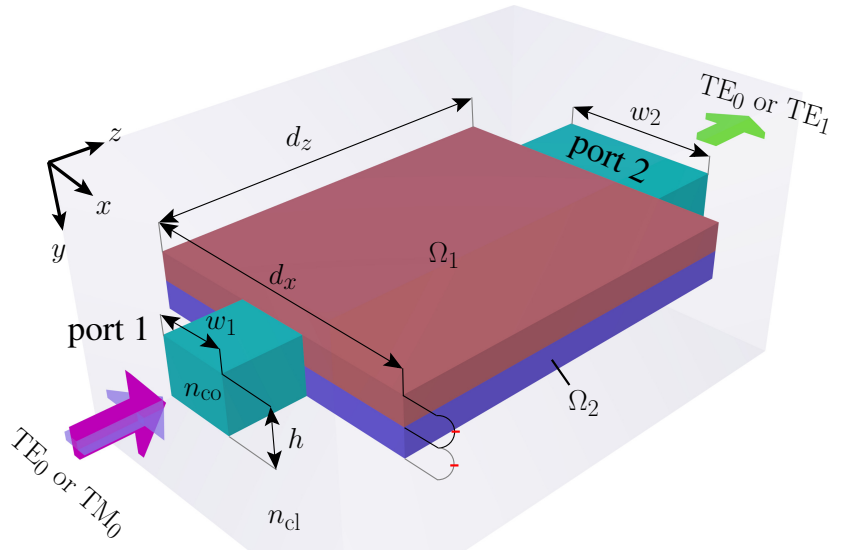

Fig. 2. A 3-D schematic of a $\mathrm{TM}_{0}$-to-TE 1 mode converter. $\Omega_{i}(i=1,2)$ is a design region.
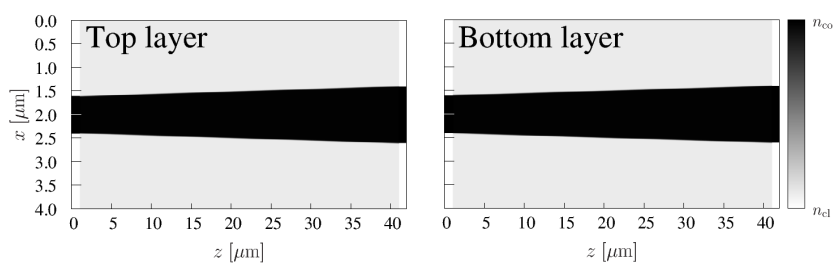

Fig. 3. An initial profile in an optimal design of a $\mathrm{TM}_{0}-$-to- $^{-\mathrm{TE}_{1}}$ mode converter.

The procedure of shape and topology optimization in this study is as follows:

1. Initialize $m, \rho_{\Omega}$, and let iteration count $M \leftarrow 0$.

2. Solve the minimization problem of objective function $f\left(\left|S_{21}\right|^{2}, \cdots,\left|S_{n 1}\right|^{2}\right)$ by repeating the following procedure until $m>m_{\max }$.

(a) Evaluate device performance using FV-BPM and compute $f$.

(b) Compute sensitivity and filter it using (19), then update $\rho_{\Omega}$ using a gradient method so that the value of $f$ decreases.

(c) If $M>0$ and $\left|f_{M}-f_{M-1}\right|<\delta$ (arbitrary small value), then let $m \leftarrow K m$ where $K(>1)$ is an arbitrary constant value.

(d) Let $M \leftarrow M+1$.

\section{APPLICATION EXAMPLE}

\section{A. $T M_{0}-t o-T E_{1}$ mode converter}

First, we apply our design approach to a design of a $\mathrm{TM}_{0^{-}}$ to- $\mathrm{TE}_{1}$ mode converter so as to illustrate its applicability. This mode converter is employed as a functional section of a polarization beam splitter and rotator (PSR) [26]-[28]. In this converter, a fundamental TE-like wave $\left(\mathrm{TE}_{0}\right)$ goes through it, and a fundamental TM-like wave $\left(\mathrm{TM}_{0}\right)$ is transformed into a first higher-order TE-like wave $\left(\mathrm{TE}_{1}\right)$. A design schematic of the mode converter is shown in Fig. 2. Core size, dimensions of a design region, and refractive indices of core and cladding

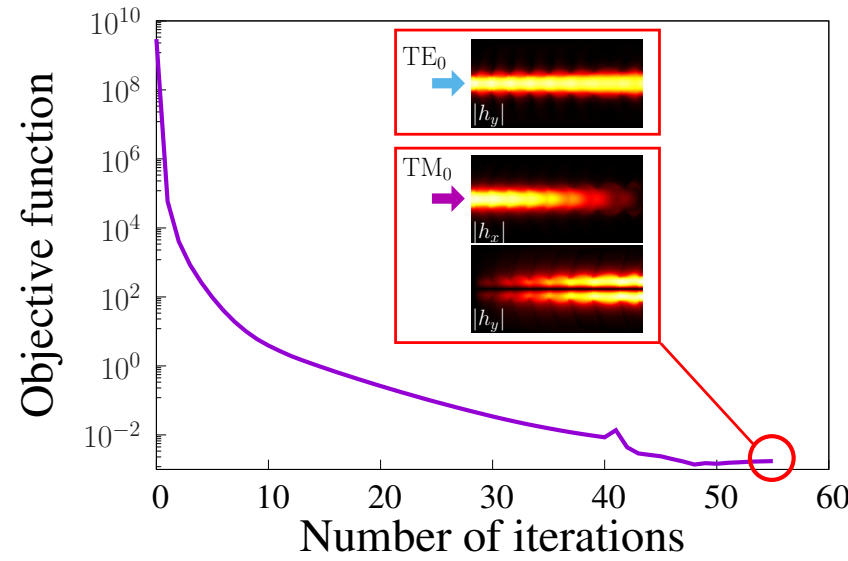

Fig. 4. Objective function as a function of iteration number in the design of a $\mathrm{TM}_{0}$-to-TE 1 mode converter. The insets show $\left|h_{x}\right|$ and $\left|h_{y}\right|$ fields calculated by the FV-BPM in an optimized structure.
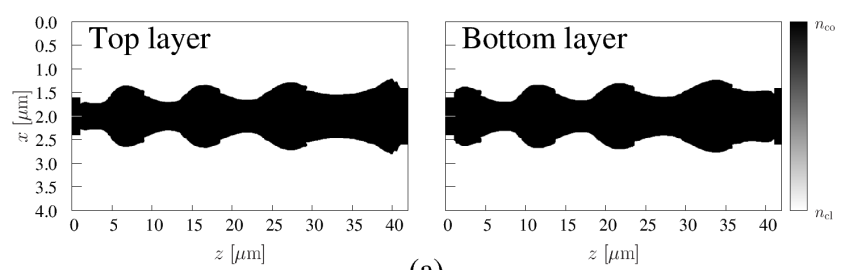

(a)

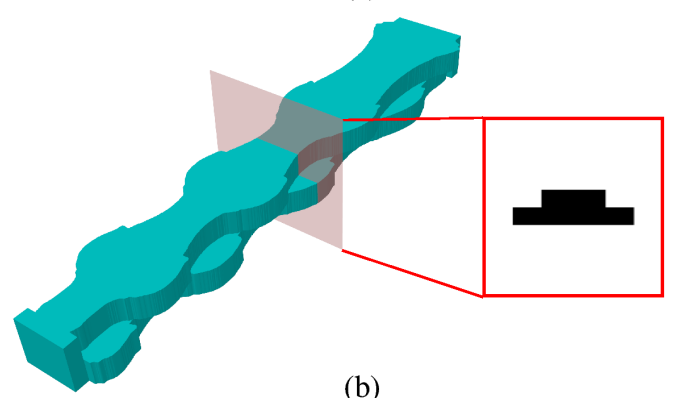

(b)

Fig. 5. An optimized profile of a $\mathrm{TM}_{0}$-to-TE 1 mode converter. (a) An optimized profile. (b) The bird's-eye view.

materials are as follows: $w_{1}=0.8 \mu \mathrm{m}, w_{2}=1.2 \mu \mathrm{m}$, $h=0.4 \mu \mathrm{m}, d_{x}=3 \mu \mathrm{m}, d_{z}=40 \mu \mathrm{m}, n_{\mathrm{co}}=2.2, n_{\mathrm{cl}}=$ 1.445. Since waveguide symmetry has to be broken vertically to induce $\mathrm{TM}_{0}$-to- $\mathrm{TE}_{1}$ conversion [26], the design region is divided into two region: $\Omega_{1}$ and $\Omega_{2}$. Density distribution in top and bottom layers, $\rho_{\Omega_{1}}$ and $\rho_{\Omega_{2}}$, are optimized simultaneously. We solve a minimization problem of the following objective function:

$$
\underset{\{\rho\}_{\Omega_{1}},\{\rho\}_{\Omega_{2}}}{\operatorname{minimize}} \quad f=f_{1}+f_{2}+f_{3}
$$

with

$$
\begin{aligned}
& f_{1}=\left(1-\left|S_{21, \mathrm{TE}_{0} \rightarrow \mathrm{TE}_{0}}\right|^{2}\right)^{2} \\
& f_{2}=\left(1-\left|S_{21, \mathrm{TM}_{0} \rightarrow \mathrm{TE}_{1}}\right|^{2}\right)^{2} \\
& f_{3}=\left(0-\frac{\left|S_{21, \mathrm{TM}_{0} \rightarrow \mathrm{TM}_{0}}\right|^{2}}{\left|S_{21, \mathrm{TM}_{0} \rightarrow \mathrm{TE}_{1}}\right|^{2}}\right)^{2}
\end{aligned}
$$




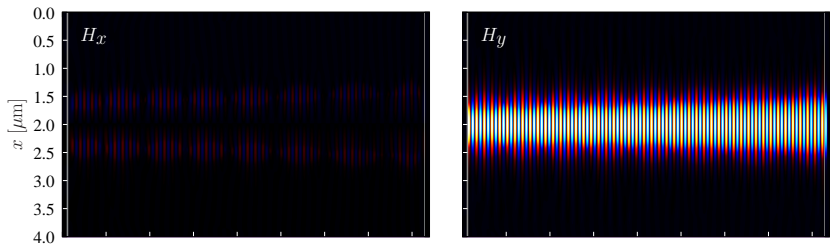

(a)

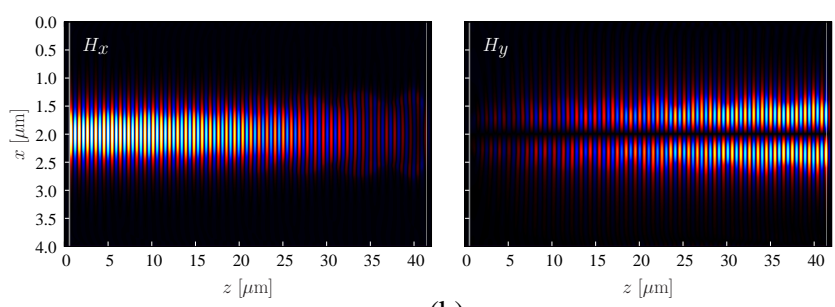

(b)

Fig. 6. Magnetic fields calculated by the FDTD method in the optimized $\mathrm{TM}_{0}$-to- $\mathrm{TE}_{1}$ mode converter: (a) $\mathrm{TE}_{0}$ wave and (b) $\mathrm{TM}_{0}$ wave at wavelength of $1.55 \mu \mathrm{m}$ are launched into port 1 .

where $\{\rho\}_{\Omega_{i}}(i=1,2)$ is a vector of density parameters. Subscripts of an S-parameter, $\mathrm{X} \rightarrow \mathrm{Y}$, denotes an input mode $(\mathrm{X})$ and an output one (Y). We estimate these S-parameters at the wavelength of $1.55 \mu \mathrm{m}$. The step size in the BPM analysis is $\Delta x=\Delta y=10 \mathrm{~nm}$ and $\Delta z=50 \mathrm{~nm}$. The transverse computational window is truncated by the PML with thickness of $0.5 \mu \mathrm{m}$. To avoid $\mathrm{TE}_{0}$-to- $\mathrm{TM}_{0}$ conversion completely, we impose symmetric condition at $x=L_{x} / 2$ where $L_{x}$ is computational window size in $x$-direction. An inverse taper waveguide shown in Fig. 3 is given as an initial profile.

The value of objective function as a function of iteration number is shown in Fig. 4. As optimization proceeds, the value of the objective function decreases monotonically until iteration count 40 . The value increases partially in iteration count from 41 to 55 because gray region is removed by increasing the value of penalty parameter $m$. Figure 5 shows an optimized profile of the mode order converter. For validation of the results of optimization with the FV-BPM, we analyze the optimized structure using the 3D-FDTD simulation. Magnetic fields calculated by a handmade 3D-FDTD solver are shown in Fig. 6. Also in the FDTD results, the $\mathrm{TE}_{0}$ wave passes through the device, and the $\mathrm{TM}_{0}$ wave is gradually transformed into the $\mathrm{TE}_{1}$ wave. Since the optimized converter is composed of periodic rib waveguides, we can see that this conversion is based on repeated re-coupling of two hybrid modes [18]. For further investigation, tolerance with respect to boundary deviation in $x$-direction, $\Delta$, is calculated using the FDTD, and the results are compared with those of the FV-BPM analysis in Fig. 7(a). One FV-BPM analysis run takes $\sim 1$ minute whereas one 3D-FDTD run takes $\sim 28$ hours using our handmade solvers and a PC with a Intel Xeon CPU E5-2660 (2.00 GHz). Although the results by the FV-BPM are not in complete agreement with those calculated by the FDTD, the tendency is almost in accord. In the FDTD simulation, the optimized profile with $+5 \mathrm{~nm}$ boundary deviation has better performance. According to the FDTD calculation, the optimized converter

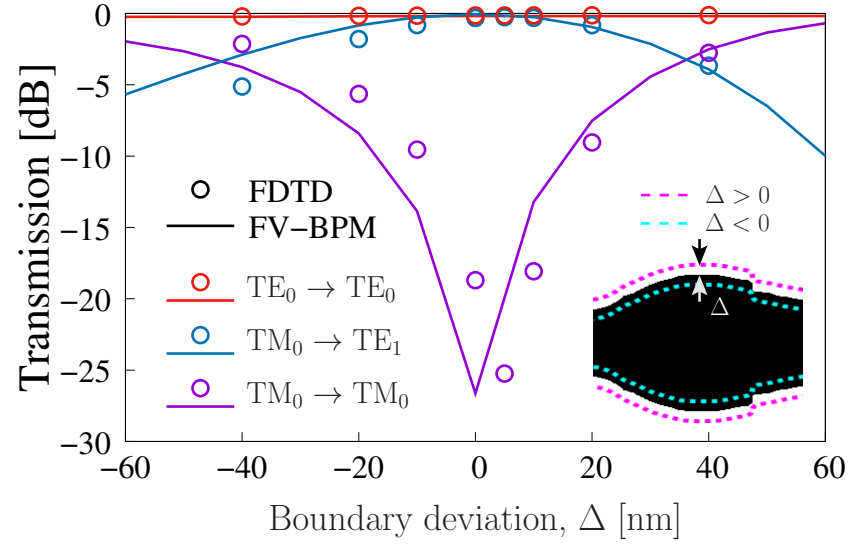

(a)

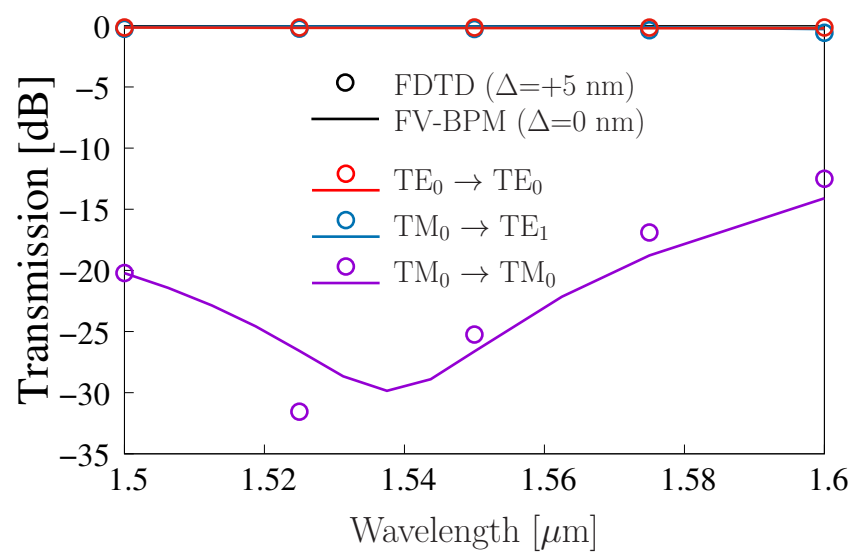

(b)

Fig. 7. (a) Tolerance with respect to boundary deviation, $\Delta$, at wavelength of $1.55 \mu \mathrm{m}$ and (b) transmission spectra in the optimized mode order converter. In (b), we compare numerical results of the FDTD $(\Delta=+5 \mathrm{~nm})$ with those of the FV-BPM $(\Delta=0 \mathrm{~nm})$.

has insertion loss of $<0.146 \mathrm{~dB}(<0.317 \mathrm{~dB})$ for $\mathrm{TE}_{0}\left(\mathrm{TM}_{0}\right)$ input, and crosstalk $\left(\mathrm{TM}_{0}\right.$-to- $\left.\mathrm{TM}_{0}\right)$ of $<-18 \mathrm{~dB}$ within the boundary deviation of $\pm 5 \mathrm{~nm}$.

Figure 7(b) shows transmission spectra and a comparison of numerical results of the FV-BPM $(\Delta=0 \mathrm{~nm})$ and the FDTD $(\Delta=+5 \mathrm{~nm})$. The FDTD results show that this converter has insertion loss of $<0.4 \mathrm{~dB}$, and crosstalk of $<-20 \mathrm{~dB}$ in the range from 1.5 to $1.565 \mu \mathrm{m}$ covering C-band. Reflection is not illustrated in the figure, but it is not significant and is at most $-30 \mathrm{~dB}$.

\section{B. Polarization rotator}

Next, we will show the design results of a PR. The configuration of a rotator is shown in Fig. 8. Core size and dimensions of a design region are as follows: $w=h=0.8 \mu \mathrm{m}$, $d_{x}=3 \mu \mathrm{m}, d_{z}=25 \mu \mathrm{m}$. In this design, dispersive nature of materials is taken into account. Core $\left(n_{\mathrm{co}}\right)$ and cladding $\left(n_{\mathrm{cl}}\right)$ indices are determined by Sellmeier equations of $\mathrm{SiO}_{2}$ [35] and $\mathrm{Si}_{3} \mathrm{~N}_{4}$ [36]. It is known that polarization rotation is induced when a cross sectional core profile is L-shaped [21]. If a core profile is simple L-shaped, two hybrid modes are 


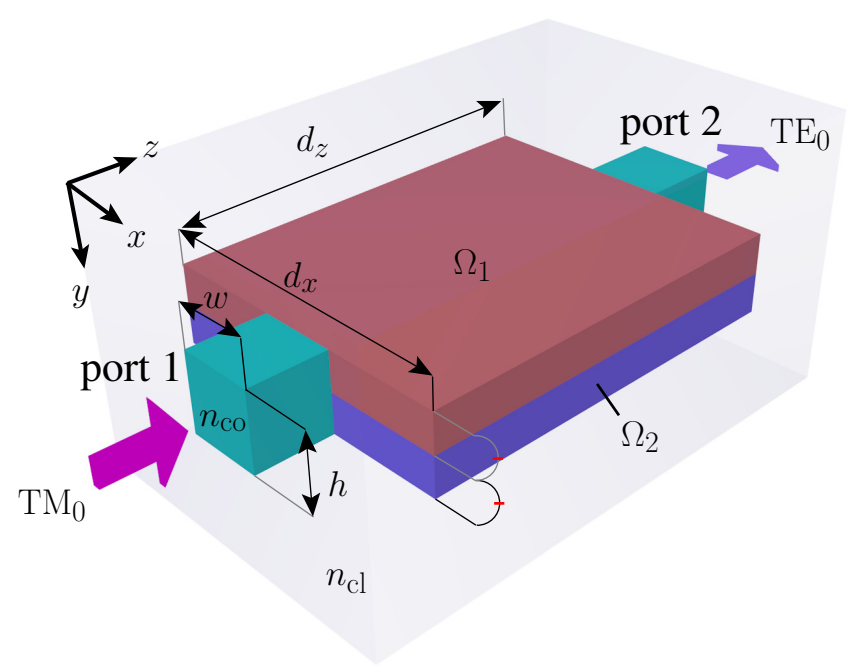

Fig. 8. A 3-D schematic view of a polarization rotator. $\Omega_{i}(i=1,2)$ is a design region.
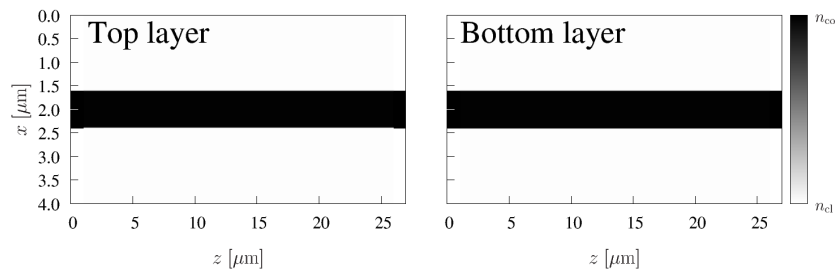

Fig. 9. An initial profile in an optimal design of a polarization rotator.

excited, and the device length can be determined by the beat length

$$
L_{\pi}=\frac{\lambda}{2 \Delta n_{e}}
$$

where $\lambda$ is wavelength and $\Delta n_{e}$ is difference of effective indices between the two hybrid modes. In a simple Lshaped rotator, $L_{\pi}$ is approximately $37.6 \mu \mathrm{m}$ at wavelength of $1.55 \mu \mathrm{m} . d_{z}$ is set to be shorter than the device length of simple one. Objective function in this design are determined as follows so that $\mathrm{TM}_{0}$ input into port 1 converts into $\mathrm{TE}_{0}$ wave at port 2 :

$$
\underset{\{\rho\}_{\Omega_{1}},\{\rho\}_{\Omega_{2}}}{\operatorname{minimize}} \quad f=\sum_{\lambda \in \Lambda}\left(f_{1}+f_{2}\right)
$$

with

$$
\begin{aligned}
& f_{1}=\left(1-\left|S_{21, \mathrm{TM}_{0} \rightarrow \mathrm{TE}_{0}}\right|^{2}\right)^{2} \\
& f_{2}=\left(0-\frac{\left|S_{21, \mathrm{TM}_{0} \rightarrow \mathrm{TM}_{0}}\right|^{2}}{\left|S_{21, \mathrm{TM}_{0} \rightarrow \mathrm{TE}_{0}}\right|^{2}}\right)^{2} .
\end{aligned}
$$

To obtain a wavelength-flattened rotator over the range from $1.5 \mu \mathrm{m}$ to $1.6 \mu \mathrm{m}$, the device is optimized at three wavelengths: $\Lambda=\{1.5 \mu \mathrm{m}, 1.55 \mu \mathrm{m}, 1.6 \mu \mathrm{m}\}$. Considering the operation principle of an L-shaped rotator, we optimize a rotator imposing symmetric condition at $z=L_{z} / 2$ where $L_{z}$

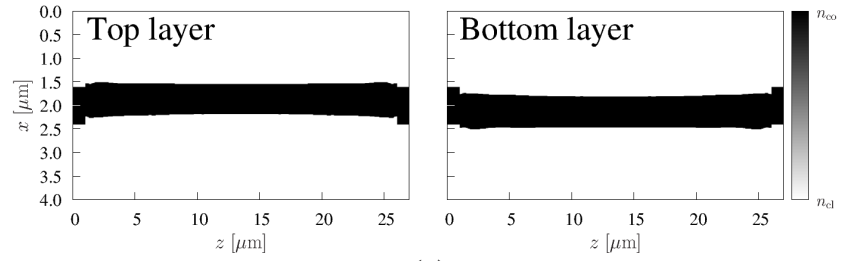

(a)

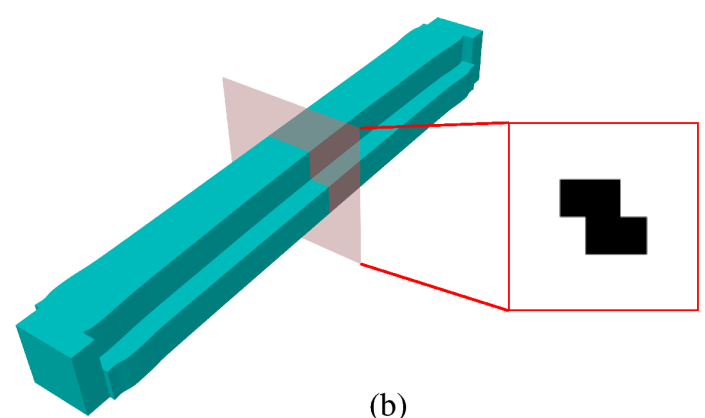

Fig. 10. An optimized polarization rotator. (a) An optimized profile. (b) The bird's-eye view.
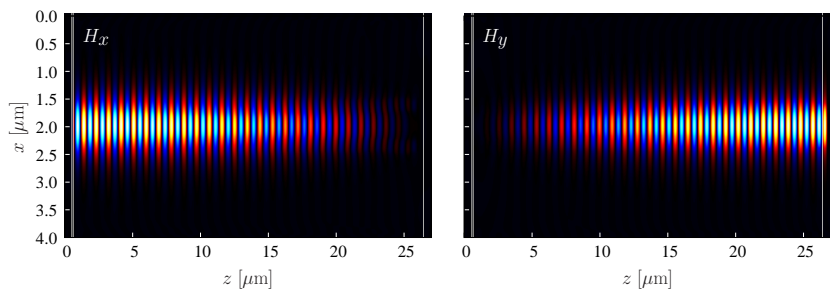

Fig. 11. Magnetic fields calculated by the FDTD method in the optimized rotator: $H_{x}$ (left) and $H_{y}$ (right) fields at wavelength of $1.55 \mu \mathrm{m}$.

is computational window size in $z$-direction. An initial profile is a straight waveguide shown in Fig. 9.

The optimized polarization rotator is shown in Fig. 10. Figure 11 shows magnetic fields calculated by the 3D-FDTD solver at wavelength of $1.55 \mu \mathrm{m}$ in the optimized rotator. We can see that almost complete polarization rotation is verified in the optimized PR. Tolerance with respect to boundary deviation and transmission spectra are calculated using the FDTD, and comparison with the results of the FV-BPM analysis is shown in Fig. 12. The results by the FV-BPM are in almost agreement with those by the FDTD, and the tendency of transmittance is practically estimated using the FV-BPM. Figure 12(a) shows tolerance with respect to $\Delta$ at a center wavelength of $1.55 \mu \mathrm{m}$. According to the FDTD simulation, insertion loss of $<0.082 \mathrm{~dB}$ and crosstalk of $<-22.4 \mathrm{~dB}$ in the range of $\Delta=[-10 \mathrm{~nm},+10 \mathrm{~nm}]$ are achieved. Figure 12(b) indicates transmission spectra of $\mathrm{TE}_{0}$ and $\mathrm{TM}_{0}$ waves at port 2. The optimized PR attains low insertion loss of $<0.06$ $\mathrm{dB}$ and low crosstalk of $<-34 \mathrm{~dB}$ in the shown range.

\section{Discussion}

As shown in the two design examples, our design approach can practically estimate shape- or topology-optimized profile although it does not always offer rigorous solution. Since 


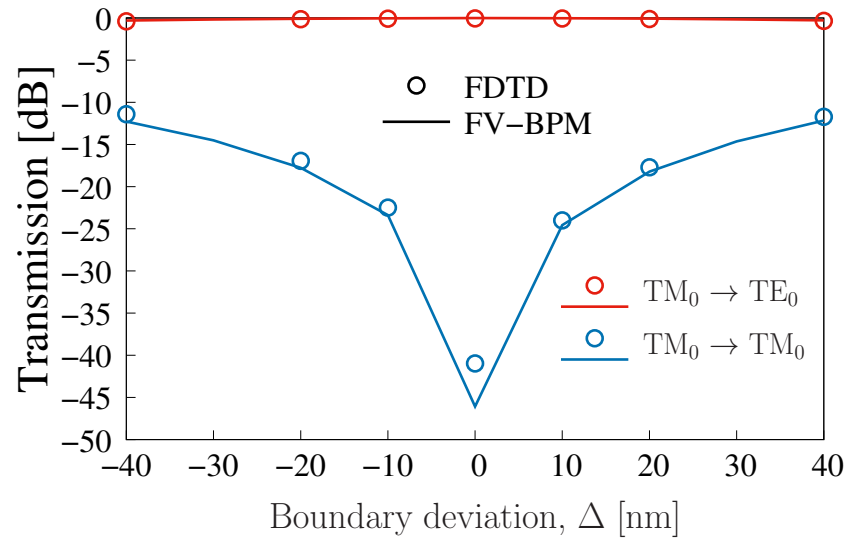

(a)

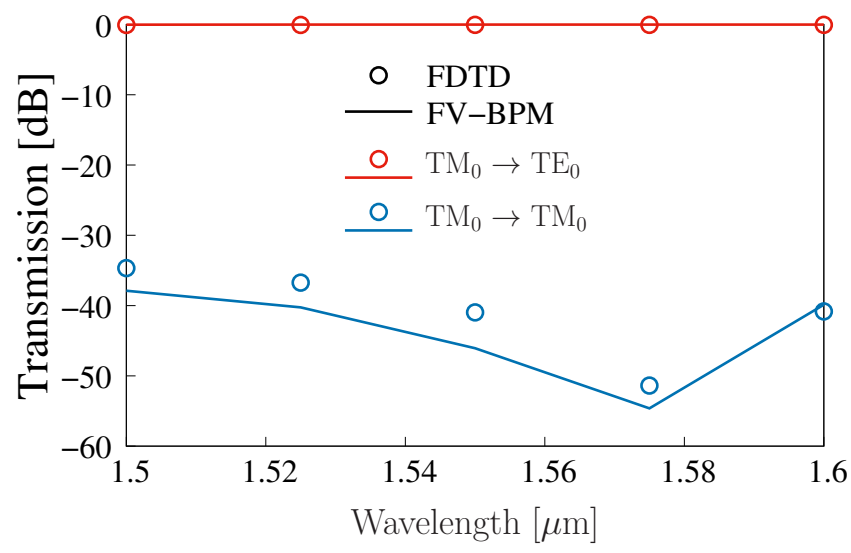

(b)

Fig. 12. Tolerance with respect to boundary deviation and transmission spectra in the optimized rotator. (b) Tolerance at a center wavelength of $1.55 \mu \mathrm{m}$ and (b) transmission spectra.

the BPM is based on the SVEA, it is difficult to apply this design approach to design of optical components which have complicated profile, such as devices based on a binary material reported in [37]-[39]. The application range of our design approach is limited to optical components where geometrical profile is gradually changed in the propagation direction. Our design approach can contribute to enhance performance of optical devices based on directional coupling, or gradual mode evolution. Recently, waveguide devices with relatively high- $\Delta$ ( $\Delta \sim 20 \%$ ) have been attracted attention because fabrication requirements can be relaxed, but these devices have a large footprint, and are time-consuming to optimize numerically using rigorous simulation methods. Since the FV-BPM costs considerably lower computational time than the FDTD, our approach is useful for efficient initial design of such optical components based on gradual mode conversion. Nevertheless, a valid geometrical constraint condition has to be studied. Although the sensitivity smoothing filter used in this work can eliminate extremely complex structure, it does not always assure appearance of specific profile which can be analyzed accurately to some extent using the FV-BPM. In the BPM, an equivalent propagation constant, $\beta_{e}$, is widely utilized so as to minimize the phase error, where $\beta_{e}$ is defined by

$$
\beta_{e}=\sqrt{\frac{\left\{\Phi_{t}\right\}^{\dagger}\left([P]-k_{0}^{2} n^{2}[I]\right)\left\{\Phi_{t}\right\}}{\left\{\Phi_{t}\right\}^{\dagger}\left\{\Phi_{t}\right\}}} .
$$

Since $\beta_{e}$ is a weighted average on phase constants of all propagating modes, it may be utilized as a constraint condition to obtain optical devices based on gradual mode evolution. It is our future work to develop a valid filtering technique and a constraint condition which forbid abrupt change of waveguide profile or $\beta_{e}$ in the propagation direction.

\section{CONCLUSION}

We presented the shape and topology optimal design approach utilizing FV-BPM for optical waveguide devices. Sensitivity analysis and design procedure are described in the specific case that the density method and the FVFD-BPM are employed. Applying our approach to design of a $\mathrm{TM}_{0}$-to-TE mode converter and a PR in the relatively high- $\Delta$ platform, we investigated the applicability of our design approach. The design results indicate that our approach has potential to be useful for efficient initial design of photonic devices based on gradual polarization rotation, or mode evolution.

\section{REFERENCES}

[1] J. S. Jensen and O. Sigmund, "Systematic design of photonic crystal structures using topology optimization: Low-loss waveguide bends," Appl. Phys. Lett., vol. 84, no. 12, pp. 2022-2024, Mar. 2004.

[2] P. I. Borel, A. Harpøth, L. H. Frandsen, M. Kristensen, P. Shi, J. S. Jensen, and O. Sigmund, "Topology optimization and fabrication of photonic crystal structures," Opt. Express, vol. 12, no. 9, pp. 1996-2001, May 2004.

[3] J. S. Jensen, O. Sigmund, L. H. Frandsen, P. I. Borel, A. Harpøth, and M. Kristensen, "Topology design and fabrication of an efficient double 90 photonic crystal waveguide bend," IEEE Photon. Technol. Lett., vol. 17, no. 6, pp. 1202-1204, June 2005.

[4] P. I. Borel, L. H. Frandsen, A. Harpøth, M. Kristensen, J. S. Jensen, and O. Sigmund, "Topology optimised broadband photonic crystal Y-splitter," Electron. Lett., vol. 41, no. 2, pp. 69-71, Jan. 2005.

[5] Y. Tsuji, K. Hirayama, T. Nomura, K. Sato, S. Nishiwaki, "Design of optical circuit devices based on topology optimization," IEEE Photon. Technol. Lett., vol. 18, no. 7, pp. 850-852, Apr. 2006.

[6] Y. Tsuji and K. Hirayama, "Design of optical circuit devices using topology optimization method with function-expansion-based refractive index distribution," IEEE Photon. Technol. Lett., vol. 20, no. 12, pp. 982 984, June 2008.

[7] T. Yasui, Y. Tsuji, J. Sugisaka, and K. Hirayama, "Design of threedimensional optical circuit devices by using topology optimization method with function-expansion-based refractive index distribution," $J$. Lightw. Technol., vol. 31, no. 23, pp. 3765-3770, Dec. 2013.

[8] R. Matzen, J. S. Jensen, and O. Sigmund, "Systematic design of slowlight photonic waveguides," J. Opt. Soc. Amer. B, vol. 28, no. 10, pp. 2374-2382, Oct. 2011

[9] L. H. Frandsen, Y. Elesin, L. F. Frellsen, M. Mitrovic, Y. Ding, O. Sigmund, and K. Yvind, "Topology optimized mode conversion in a photonic crystal waveguide fabricated in silicon-on-insulator material," Opt. Express, vol. 22, no. 7, pp. 8525-8532, Apr. 2014.

[10] J. Lu and J. Vučković, "Nanophotonic computational design," Opt. Express, vol. 21, no. 11, pp. 13351-13367, June 2013.

[11] M. D. Feit and J. A. Fleck, "Light propagation in graded-index optical fibers," Appl. Opt., vol. 17, no. 24, pp. 3990-3998, Dec. 1978.

[12] Y. Chung and N. Dagli, "An assessment of finite difference beam propagation method," IEEE J. Quantum. Electron., vol. 26, no. 8, pp. 1335-1339, Aug. 1990.

[13] W. P. Huang and C. L. Xu, "Simulation of three-dimensional optical waveguides by a full-vector beam propagation method," IEEE J. Quantum. Electron., vol. 29, no. 10, pp. 2639-2649, Oct. 1993. 
[14] J. Yamauchi, G. Takahashi, and H. Nakano, "Full-vectorial beampropagation method based on the McKee-Mitchell scheme with improved finite-difference formulas," J. Ligthw. Technol., vol. 16, no. 12, pp. 24582464, Dec. 1998.

[15] Y. Hsueh, M. Yang, and H. Chang, "Three-dimensional noniterative fullvectorial beam propagation method based on the alternating direction implicit method," J. Ligthw. Technol., vol. 17, no. 11, pp. 2389-2397, Nov. 1999.

[16] A. Iguchi, Y. Tsuji, T. Yasui, and K. Hirayama, "Topology optimization of optical waveguide devices based on beam propagation method with sensitivity analysis," J. Ligthw. Technol., vol. 34, no. 18, pp. 4214-4220, Sept. 2016.

[17] A. Iguchi, Y. Tsuji, T. Yasui, and K. Hirayama, "Efficient topology optimization of optical waveguide devices utilizing semi-vectorial finitedifference beam propagation method," Opt. Express, vol. 25, no. 3, pp. 28210-28222, Nov. 2017.

[18] W. Huang and Z. M. Mao, "Polarization rotation in periodic loaded rib waveguides," J. Ligthw. Technol., vol. 10, no. 12, pp. 1825-1831, Dec. 1992.

[19] M. R. Watts and H. A. Haus, "Integrated mode-evolution-based polarization rotators," Opt. Lett., vol. 30, no. 2, pp. 138-140, Jan. 2005.

[20] H. Fukuda, K. Yamada, T. Tsuchizawa, T. Watanabe, H. Shinojima, and S. Itabashi, "Polarization rotator based on silicon wire waveguides," Opt. Express, vol. 16, no. 4, pp. 2628-2635, Feb. 2008.

[21] Z. Wang and D. Dai, "Ultrasmall Si-nanowire-based polarization rotator," J. Opt. Soc. Amer. B, vol. 25, no. 5, pp. 747-753, May. 2008.

[22] X. Sun, M. Z. Alam, J. S. Aitchison, and M. Mojahedi, "Polarization rotator based on augmented low-index-guiding waveguide on silicon nitride/silicon-on-insulator platform," Opt. Lett., vol. 41, no. 14, pp. 32293232, July 2016

[23] C. Sun, Y. Yu, G. Chen, and X. Zhang, "A low crosstalk and broadband polarization rotator and splitter based on adiabatic couplers," IEEE Photon. Technol. Lett., vol. 28, no. 20, pp. 2253-2256, Oct. 2016.

[24] H.-C. Chung and S.-Y. Tseng, "Ultrashort and broadband silicon polarization splitter-rotator using fast quasiadiabatic dynamics," Opt. Express, vol. 26, no. 8, pp. 9655-9665, Apr. 2018.

[25] S. Guerber, C. A.-Ramos, D. Benedikovic, E. D.-Valdeiglesias, X. L. Roux, N. Vulliet, E. Cassan, D. M.-Morini, C. Baudot, F. Boeuf , and L. Vivien, "Broadband polarization beam splitter on a silicon nitride platform for O-band operation," IEEE Photon. Technol. Lett., vol. 30, no. 19, pp. 1679-1682, Oct. 2018.

[26] D. Dai and J. E. Bowers, "Novel concept for ultracompact polarization splitter-rotator based on silicon nanowires," Opt. Express, vol. 19, no. 11, pp. 10940-10949, May 2011.

[27] W. D. Sacher, T. Barwicz, B. J. F. Taylor, and J. K. S. Poon, "Polarization rotator-splitters in standard active silicon photonics platforms," Opt. Express, vol. 22, no. 4, pp. 3777-3786, Feb. 2014.

[28] J. Wang, B. Niu, Z. Sheng, A. Wu, W. Li, X. Wang, S. Zou, M. Qi, and F. Gan, "Novel ultra-broadband polarization splitter-rotator based on mode-evolution tapers and a mode-sorting asymmetric Y-junction," Opt. Express, vol. 22, no. 11, pp. 13565-13571, June 2014.

[29] M. A. Swillam, M. H. Bakr, and X. Li, "Full vectorial 3-D sensitivity analysis and design optimization using BPM," J. Lightw. Technol., vol. 26, no. 5, pp. 528-536, Mar. 2008.

[30] M. S. Stern, "Semivectorial polarised finite difference method for optical waveguides with arbitrary index profiles," IEE Proc. J, Vol. 135, No. 1, pp. 56-63, Feb. 1988.

[31] M. S. Stern, "Semivectorial polarised H field solutions for dielectric waveguides with arbitrary index profiles," IEE Proc. J, Vol. 135, No. 5, pp. 333-338, Oct. 1988.

[32] Y. He and F.G. Shi, "Improved full-vectorial beam propagation method with high accuracy for arbitrary optical waveguides," IEEE Photon. Technol. Lett., Vol. 15, No. 10, pp. 1381-1383, Sept. 2003.

[33] J. Xiao and X. Sun, "A Modified full-vectorial finite-difference beam propagation method based on $\mathrm{H}$-fields for optical waveguides with stepindex profiles," Opt. Commun., Vol. 266, No. 2, pp. 505-511, Oct. 2006.

[34] W. C. Chew and W. H. Weedon, "A 3-D perfectly matched medium from modified Maxwell's equations with stretched coordinates," Micro. Opt. Technol. Lett., Vol. 7, No. 13, pp. 599-604, Sept. 1994.

[35] C. Z. Tan, "Determination of refractive index of silica glass for infrared wavelengths by IR spectroscopy," J. Non-Cryst. Solids, vol. 223, pp. 158163, Jan. 1998.

[36] K. Luke , Y. Okawachi, M. R. E. Lamont, A. L. Gaeta, and M. Lipson, "Broadband mid-infrared frequency comb generation in a $\mathrm{Si}_{3} \mathrm{~N}_{4}$ microresonator," Opt. Lett., vol. 21, no. 21, pp. 4823-4826, Nov. 2015.
[37] B. Shen, P. Wang, R. Polson, and R. Menon, "An integratednanophotonics polarization beamsplitter with $2.4 \times 2.4 \mu \mathrm{m}^{2}$ footprint," Nature Photon., vol. 9, pp. 378-382, May 2015.

[38] W. Chang, L. Lu, X. Ren, D. Li, Z. Pan, M. Cheng, D. Liu, and M. Zhang, "Ultra-compact mode (de) multiplexer based on subwavelength asymmetric Y-junction," vol. 26, no. 7, pp. 8162-8170, Mar. 2018.

[39] W. Chang, Y. Ao, L. Lu, S. Fu, L. Deng, M. Cheng, L. Xia, D. Liu, and M. Zhang, "Inverse design and demonstration of ultracompact silicon polarization rotator," Opt. Fiber Commun. Conf. Expo. (OFC), W3B.2, 2019.

Akito Iguchi (S'15-M'19) received the B.S., M.S., and Ph.D. degrees in electronic engineering from Muroran Institute of Technology, Muroran, Japan, in 2015,2017 , and 2019.

$\mathrm{He}$ is presently a post-doctoral research fellow of Japan Society for the Promotion of Science (JSPS), and is a research member of Wave Electronics Laboratory at Muroran Institute of Technology.

Dr. Iguchi is a member of the Institute of Electronics, Information and Communication Engineers (IEICE) and IEEE.

Yasuhide Tsuji (M'97-SM'19) received the B.S., M.S., and Ph.D. degrees in electronic engineering from Hokkaido University, Sapporo, Japan, in 1991, 1993, and 1996, respectively.

In 1996, he joined the Department of Applied Electronic Engineering, Hokkaido Institute of Technology, Sapporo, Japan. From 1997 to 2004, he was an Associate Professor of Electronics and Information Engineering at Hokkaido University. From 2004 to 2011, he was an Associate Professor of Electrical and Electronic Engineering at Kitami Institute of Technology, Kitami, Japan. Since 2011, he has been a professor of Information and Electronic Engineering at Muroran Institute of Technology, Muroran, Japan. $\mathrm{He}$ has been interested in wave electronics.

Dr. Tsuji is a member of the Institute of Electronics, Information and Communication Engineers (IEICE), the Japan Society of Applied Physics, the Optical Society of America (OSA), and a Senior member of IEEE. In 1997, 1999, and 2019, he was awarded the Best Paper Award from IEICE. In 2000, he has received the Third Millennium Medal from IEEE.

Takashi Yasui (S'00-M'02) received the B.S. degree in electronic engineering from Fukui University, Fukui, Japan, in 1997, and the M.S. and Ph.D. degrees in electronic engineering from Hokkaido University, Sapporo, Japan, in 1999 and 2001, respectively.

From 1999 to 2002, he was a Research Fellow of the Japan Society for the Promotion of Science. In 2002, he joined Fujitsu Ltd., Chiba, Japan. From 2004 to 2011, he was an Assistant Professor of the Department of Electronic and Control Systems Engineering, Shimane University, Matsue, Japan. Since 2011, he has been an Associate Professor of the Department of Electrical and Electronic Engineering, Kitami Institute of Technology, Kitami, Japan. He has been engaged in research on wave electronics.

Dr. Yasui is a member of the Applied Computational Electromagnetics Society, the Optical Society of America, and the Institute of Electronics, Information, and Communication Engineers of Japan. In 2018, he was awarded the Best Paper Award from IEICE.

Koichi Hirayama (SM'96) received the B.S., M.S., and Ph.D. degrees in electronic engineering from Hokkaido University, Sapporo, Japan, in 1984, 1986, and 1989, respectively.

In 1989, he joined the Department of Electronic Engineering, Kushiro National College of Technology, Kushiro, Japan. In 1992, he became an Associate Professor of Electronic Engineering at Kitami Institute of Technology, Kitami, Japan, and in 2004 he became a Professor. He has been interested in the analysis and optimal design of electromagnetic and optical waveguides.

Dr. Hirayama is a member of the Institute of of Electronics, Information and Communication Engineers (IEICE) of Japan and the Japan Society of Applied Physics. In 2018, he was awarded the Best Paper Award from IEICE. 